\title{
Excessive double strange baryon production due to strangeness oscillation in $p+A, A+A$ collisions
}

\section{Peter Filip* ${ }^{* \dagger}$}

Institute of Physics, Slovak Academy of Sciences, Dúbravská cesta 9, Bratislava 845 11, Slovakia

E-mail: fyziflipesavba.sk

Production of double strange $\Xi^{-}$hyperons at sub-threshold energies has been observed by HADES experiment [1] to be unexpectedly enhanced in comparison to theoretical estimates. We suggest, that $K^{0} \leftrightarrow \bar{K}^{0}$ oscillation of neutral kaons can be affected in very dense baryonic matter in a specific way, which may result in the oscillation length 5-10 fm. This allows for the strangeness violation process $(\bar{s} d) \rightarrow(s \bar{d})$ to occur in a very short time, within the volume of dense hadronic medium, and excessive double strange hyperons can be created via rescattering $\bar{K}^{0}+\left(\Sigma^{0}, \Lambda\right) \rightarrow \Xi+\pi$ interactions. The significance of such processes is underestimated, if global strangeness conservation is assumed in $p+A$ and $A+A$ collisions at low energies.

38th International Conference on High Energy Physics

3-10 August 2016

Chicago, USA

*Speaker.

${ }^{\dagger}$ Support from ICHEP organizers and from Slovak Grant Agency VEGA (project 2/197/14) is acknowledged. 


\section{Introduction}

The oscillation of neutral mesons $\left(K^{0} \leftrightarrow \bar{K}^{0}\right)$ is a beautiful quantum mechanical phenomenon, which allows [2] us to study also the fundamental properties of nature (CP symmetry). $B_{d}^{0} \leftrightarrow \bar{B}_{d}^{0}$ and $B_{s}^{0} \leftrightarrow \bar{B}_{s}^{0}$ oscillations have been clearly observed as well [3], while $D^{0}, \bar{D}^{0}$ mesons containing only up-type quarks have been proved to oscillate only recently [4].

The period of $K^{0} \leftrightarrow \bar{K}^{0}$ transitions (oscillation length $L_{o s c}$ ) is determined by the mass difference $\Delta m_{K^{0}}=m\left(K_{2}^{0}\right)-m\left(K_{1}^{0}\right)$ of eigenstates $K_{2}^{0}$ and $K_{1}^{0}$ of the weak $\mathbb{H}_{w}$ hamiltonian. Measured values of $\Delta m_{K^{0}}, \Delta m_{D^{0}}, \Delta m_{B_{d}^{0}}$, and $\Delta m_{B_{s}^{0}}$ mass differences in vacuum are (in $10^{10} \hbar / s$ units): $0.529 \pm 0.001$, $0.95 \pm 0.44,51.0 \pm 0.3$, and $1776 \pm 2$, which gives [3] oscillation lengths $L_{o s c}=c \hbar / \Delta m: 35 \mathrm{~cm}$, $\approx 20 \mathrm{~cm}, 3.7 \mathrm{~mm}$, and $0.11 \mathrm{~mm}$. Standard Model explains these oscillations succesfully by $2^{\text {nd }}$ order flavour-changing transitions $(s \bar{d}) \leftrightarrow(\bar{s} d)$ and $(c \bar{u}) \leftrightarrow(\bar{c} u)$ and $(b \bar{d}, \bar{s}) \leftrightarrow(\bar{b} d, s)$ taking place in the vacuum due to short-distance (box diagrams) and long-distance effects [3].

In the material medium (a regenerator), or in a dense nuclear matter, the value of $\Delta m_{K^{0}}$ mass difference of $K_{2}^{0}, K_{1}^{0}$ eigenstates may become modified due to meson-baryon (repulsive) and antimeson-baryon (attractive) potentials [5]. Linear approximation (see Eq. 66 and 67 in [5])

$$
m_{K^{0}}(\rho)=\left(1+\alpha_{K} \frac{\rho}{\rho_{0}}\right) m_{K^{0}}^{\rho=0} \quad ; \quad m_{\bar{K}^{0}}(\rho)=\left(1-\tilde{\alpha}_{\bar{K}} \frac{\rho}{\rho_{0}}\right) m_{\bar{K}^{0}}^{\rho=0} .
$$

gives $m\left(K^{0}\right)-m\left(\bar{K}^{0}\right) \approx 80 \mathrm{MeV}$ at $\rho=\rho_{0}$ density, if values $\alpha_{K} \approx 0.05$ and $\tilde{\alpha}_{\bar{K}} \approx 0.12$ are used.

In this contribution we suggest $\Delta m_{K^{0}}$ mass difference in dense baryonic medium may become so large, that $(\bar{s} \rightarrow s)$ transition length $L^{\bar{s} \rightarrow s}=c \hbar / 2 \Delta m_{K^{0}}$ can be very short: $2-10 \mathrm{fm}$. This may allow for a single $(s \bar{s})$ pair (created in the low-energy $p+A$ and $A+A$ collisions) to be sufficient for the production of double strange $\Xi^{-}(s s d)$ hyperons via process:

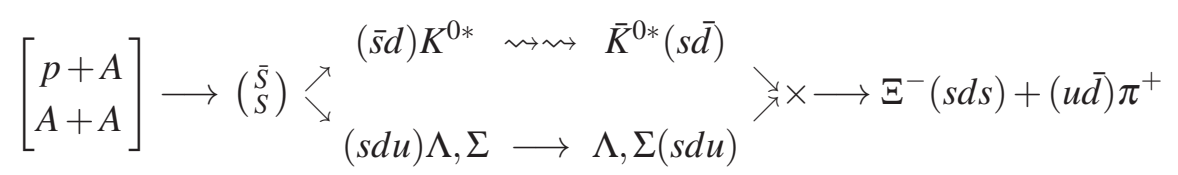

At high baryonic densities, $\bar{s}$ quarks preferentially hadronize into $K^{0}(d \bar{s})$ or $K^{+}(u \bar{s})$ mesons, while $s$ quarks are trapped into hyperons. When multiplicities of neutral $K^{0}(d \bar{s})$ and $\bar{K}^{0}(s \bar{d})$ mesons are very asymmetric (e.g. $N[K] / N[\bar{K}] \geq 100$ ), mesons $K^{0}(d \bar{s})$ may enhance $s$ quark population via fast $K^{0} \rightarrow \bar{K}^{0}$ transition. Excessive $\Xi^{-}(s s d)$ or $\Xi^{0}(s s u)$ hyperons may thus be created in $p+A$ and $A+A$ collisions at subthreshold energy [1] via rescattering process: $\bar{K}^{0}+\left(\Sigma^{0}, \Lambda\right) \rightarrow \Xi+\pi$.

\section{Neutral kaons in dense baryonic medium}

In the vacuum (if $\mathrm{CP}$ violation effects $|\varepsilon| \approx 2 \cdot 10^{-3}$ are neglected) the eigenstates $K_{1,2}^{0}$ of weak Hamiltonian $\mathbb{H}_{w}$ are: $K_{1,2}^{0}=\left(K^{0} \pm \bar{K}^{0}\right) / \sqrt{2}$. In a medium, Hamiltonian $\mathbb{H}_{w}^{\prime}=\mathbb{M}^{\prime}-\frac{i}{2} \mathbb{G}^{\prime}$ is

$$
\mathbb{H}_{w}^{\prime}=\left[\begin{array}{cc}
M_{11}+V_{K^{0}}(\rho) & M_{12} \\
M_{21} & M_{22}-\bar{V}_{\bar{K}^{0}}(\rho)
\end{array}\right]-\frac{i}{2}\left(\begin{array}{cc}
\Gamma_{11}+A_{K^{0}}(\rho) & \Gamma_{12} \\
\Gamma_{21} & \Gamma_{22}+\bar{A}_{\bar{K}^{0}}(\rho)
\end{array}\right)
$$

and linear approximation (1.1) gives potentials $V_{K^{0}}=m_{K^{0}} \alpha_{K}\left(\rho / \rho_{0}\right)$ and $\bar{V}_{\bar{K}^{0}}=m_{\bar{K}^{0}} \tilde{\alpha}_{\bar{K}}\left(\rho / \rho_{0}\right)$. This means $V_{K^{0}} \approx 20 \mathrm{MeV}$ and $\bar{V}_{\bar{K}^{0}} \approx 60 \mathrm{MeV}$ at nuclear density $\rho \approx \rho_{0}=2 \cdot 10^{17} \mathrm{~kg} / \mathrm{m}^{3}$, for (momentum 
averaged [5]) parameters $\alpha_{K} \approx 0.04$ and $\tilde{\alpha}_{\bar{K}} \approx 0.12$. Absorption coeficients $A_{K^{0}}, \bar{A}_{\bar{K}^{0}}$ in (2.1) are related to forward scattering amplitude difference $f_{K}(0)-\bar{f}_{\bar{K}}(0)$ of $K^{0}, \bar{K}^{0}$ mesons in medium [6].

Diagonalization of $2 \times 2$ non-hermitian Hamiltonian (2.1) with $M_{11}=M_{22}=497 \mathrm{MeV}$ and $\Gamma_{11}=\Gamma_{22}=3.7 \cdot 10^{-12} \mathrm{MeV}$ (using $\left|\Gamma_{12}\right|=\left|\Gamma_{21}\right| \approx 3.48 \cdot 10^{-12}$ and $\left|M_{12}\right|=\left|M_{21}\right| \approx 1.74 \cdot 10^{-12}$ ) allows to obtain difference of $K_{1,2}^{0}$ eigenstate masses and decay widths in medium [7] as

$$
\Delta \mu=\tilde{m}\left(K_{2}^{0}\right)-\tilde{m}\left(K_{1}^{0}\right)-\frac{i}{2}\left(\tilde{\Gamma}_{K_{2}^{0}}-\tilde{\Gamma}_{K_{1}^{0}}\right)=\sqrt{4 H_{12} H_{21}+\left(H_{22}-H_{11}\right)^{2}}=\Delta \tilde{m}_{K}-\frac{i}{2} \Delta \tilde{\Gamma}_{K} .
$$

Probabilities of $K^{0} \leftrightarrow \bar{K}^{0}$ transitions are (using Eq. 9.7 and 9.8 from Ref. [7])

$$
P\left[K^{0} \rightsquigarrow \bar{K}^{0}\right]=\left|\frac{q_{H}}{p_{H}}\right|^{2}\left|g_{-}(\tau)\right|^{2}|(1-\theta)|^{2}, \quad P\left[\bar{K}^{0} \rightsquigarrow K^{0}\right]=\left|\frac{p_{L}}{q_{L}}\right|^{2}\left|g_{-}(\tau)\right|^{2}|(1-\theta)|^{2},
$$

where the interference term

$$
\left|g_{-}(\tau)\right|^{2}=\frac{1}{4}\left[e^{-\tau \tilde{\Gamma}_{2}^{o}(\rho)}+e^{-\tau \tilde{\Gamma}_{K_{1}^{o}}(\rho)}-2 \cos \left[\Delta \tilde{m}_{K}(\rho) \tau\right] \cdot e^{-\tau\left[\tilde{\Gamma}_{K_{2}^{o}}(\rho)+\tilde{\Gamma}_{K_{1}^{o}}(\rho)\right] / 2}\right]
$$

in (2.3) is multiplied by $\left|q_{H} / p_{H}\right|=2\left|H_{21}\right| /|\Delta \mu(1-\theta)|$ quantity [7]. Conservation of CP symmetry gives $\left|q_{H} / p_{H}\right|=\left|p_{L} / q_{L}\right|$, and weak hamiltonian $\mathbb{H}_{w}^{\prime}$ eigenvectors are: $K_{2}^{0}=p_{H}\left|K^{0}\right\rangle+q_{H}\left|\bar{K}^{0}\right\rangle$ and $K_{1}^{0}=p_{L}\left|K^{0}\right\rangle-q_{L}\left|\bar{K}^{0}\right\rangle$. Consequently, one obtains for $K^{0} \rightsquigarrow \bar{K}^{0}$ transition probability

$$
P\left[K^{0} \rightsquigarrow \bar{K}^{0}\right]=\frac{\left|2 H_{21}\right|^{2}}{|\Delta \mu|^{2}}\left|g_{-}(\tau)\right|^{2} \approx \frac{4\left|M_{21}-\frac{i}{2} \Gamma_{21}\right|^{2}}{\left|H_{22}-H_{11}\right|^{2}}\left|g_{-}(\tau)\right|^{2}=S_{\rho}\left|g_{-}(\tau)\right|^{2}
$$

where $S_{\rho}$ is the suppression factor of $|\Delta S|=2$ process $K^{0}(d \bar{s}) \rightsquigarrow \bar{K}^{0}(s \bar{d})$ in the medium. For $\bar{K}^{0}, K^{0}(497)$ pseudoscalar mesons at nuclear density $\rho \approx \rho^{0}$, one may expect $\left|H_{22}-H_{11}\right| \approx 100 \mathrm{MeV}$. Values $\left|\Gamma_{12}\right|=\left|\Gamma_{21}\right|=3.48 \cdot 10^{-12}$ and $\left|M_{12}\right|=\left|M_{21}\right|=1.74 \cdot 10^{-12} \mathrm{MeV}$ [7] then give enormous suppression factor $S_{\rho} \leq 10^{-26}$ for $K^{0} \leftrightarrow \bar{K}^{0}$ oscillations in nuclear medium, in agreement with [8].

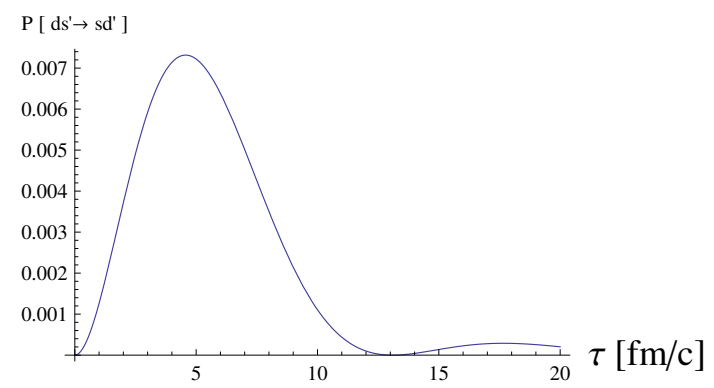

Figure 1: $K^{0 *} \rightarrow \bar{K}^{0 *}$ transition probability as a function of time $\tau$ obtained for density $\rho / \rho_{0}=1.2$.

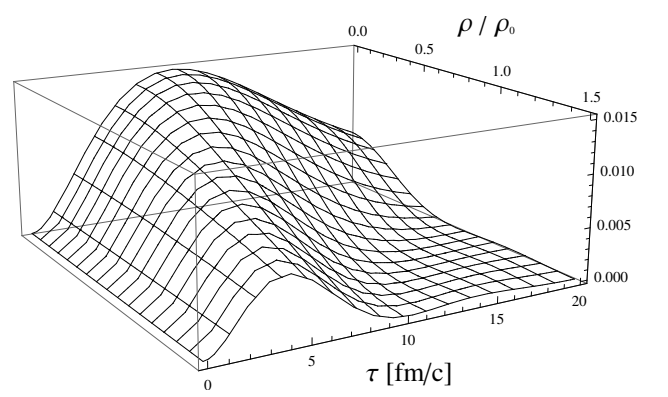

Figure 2: Probability of $K^{0 *} \rightarrow \bar{K}^{0 *}$ oscillation as a function of time and baryonic density $\rho<1.5 \rho_{0}$.

However, for $K^{0 *}(896)$ and $\bar{K}^{0 *}(896)$ mesons, which may also form weak eigenstates [9] $K_{L, S}^{0 *}=\left(K^{0 *} \pm \bar{K}^{0 *}\right) / \sqrt{2}$, one obtains $S_{\rho} \approx 10^{-2}$ (see Figures 1 and 2 ), assuming $K^{0 *}, \bar{K}^{0 *}$ mesons share $33 \%$ of their decay products $\left(K^{0 *} \rightarrow K_{S, L}^{0}+\pi^{0}\right.$ and $\left.\bar{K}^{0 *} \rightarrow K_{S, L}^{0}+\pi^{0}\right)$. Indeed, one has [7]

$$
\Gamma_{12}=\rho_{c}\left\langle K^{0 *}\left|H_{w}^{\prime}\right| K_{S}^{0} \pi^{0}\right\rangle\left\langle K_{S}^{0} \pi^{0}\left|H_{w}^{\prime}\right| \bar{K}^{0 *}\right\rangle+\rho_{c}\left\langle K^{0 *}\left|H_{w}^{\prime}\right| K_{L}^{0} \pi^{0}\right\rangle\left\langle K_{L}^{0} \pi^{0}\left|H_{w}^{\prime}\right| \bar{K}^{0 *}\right\rangle
$$


which gives $\Gamma_{12} \approx 16 \mathrm{MeV}$, for widths $\Gamma\left(K^{0 *} \rightarrow K_{L, S}^{0}+\pi^{0}\right)=\Gamma\left(\bar{K}^{0 *} \rightarrow K_{L, S}^{0}+\pi^{0}\right)=8+8 \mathrm{MeV}$. Using $\Gamma_{12}=16 \mathrm{MeV}$ and $\Gamma_{11}=\Gamma_{22}=\Gamma_{K^{*}}=48 \mathrm{MeV}$ in the Hamiltonian for $K^{0 *}, \bar{K}^{0 *}$ mesons

$$
\mathbb{H}_{K^{0 *}}^{\prime}=\left[\begin{array}{cc}
896+V_{K^{0}}(\rho) & 1.7 \cdot 10^{-12} \\
1.7 \cdot 10^{-12} & 896-\bar{V}_{\bar{K}^{0}}(\rho)
\end{array}\right]-\frac{i}{2}\left(\begin{array}{cc}
48+A_{K^{0}} & 16 \cdot e^{i \zeta} \\
16 \cdot e^{-i \zeta} & 48+\bar{A}_{\bar{K}^{0}}
\end{array}\right)
$$

suppression factor $S\left(\rho_{B}\right) \approx 10^{-2}$ is obtained in Eq.(2.5) for $\Delta V_{K^{*}}=80 \mathrm{MeV}$ at density $\rho=\rho^{0}$. In Figures 3 and 4 we show $\Delta m_{K^{*}}=m\left(K_{2}^{0 *}\right)-m\left(K_{1}^{0 *}\right)$ mass difference and $K^{0 *} \rightarrow \bar{K}^{0 *}$ transition length $L^{\bar{s} \rightarrow s}=c \cdot \tau_{\text {osc }} / 2$ evaluated for $K^{0 *}, \bar{K}^{0 *}$ mesons in baryonic matter using Hamiltonian (2.7).

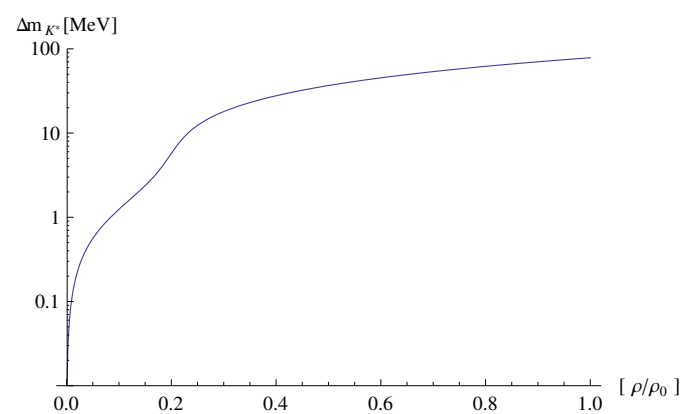

Figure 3: Mass difference $\Delta m_{K^{*}}=m\left(K_{2}^{0 *}\right)-m\left(K_{1}^{0 *}\right)$ of weak eigenstates $K_{2}^{0 *}, K_{1}^{0 *}$ in the nuclear medium.

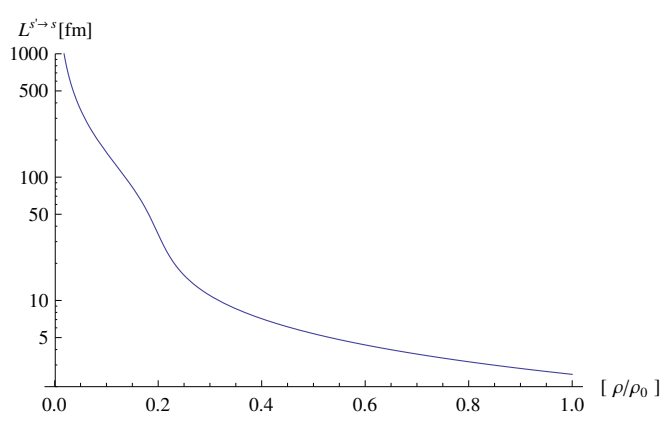

Figure 4: Dependence of $K^{0 *} \rightarrow \bar{K}^{0 *}$ transition length $L^{\bar{s} \rightarrow s}=\hbar c / 2 \Delta m_{K^{*}}$ on baryonic density $\rho / \rho_{0}$.

\section{Summary and conclusions}

We have considered $(d \bar{s}) \leftrightarrow(\bar{d} s)$ oscillations in dense nuclear matter. We suggest $K^{0 *} \rightarrow \bar{K}^{0 *}$ process may happen in $p+A$ or $A+A$ collisions [1] within time scale $(3-10 \mathrm{fm} / \mathrm{c}$ ) with probability $\approx 1 \%$. This may allow for the excessive $\Xi^{-}(s s d)$ hyperon production via $\bar{K}^{0}+\left(\Sigma^{0}, \Lambda\right) \rightarrow \Xi+\pi$ reaction at sub-threshold energies, when single $(s \bar{s})$ pair is produced. If $N\left(K^{0 *}\right) / N\left(\bar{K}^{0 *}\right) \geq 100$ condition is valid in $A+A$ collisions, $(\bar{s} / s)$ ratios may be modified due to $K^{0 *} \rightarrow \bar{K}^{0 *}$ processes. In agreement with Ref. [8] we find $K^{0} \rightarrow \bar{K}^{0}$ transitions in dense baryonic matter to be negligible.

Although fast oscillations of $K^{0}, B_{s}^{0}, B^{0}$ mesons in the nuclear medium are unlikely, a modification of $\Delta \tilde{m}_{K}$ and $\Delta \tilde{m}_{B}$ parameters in dense regenerators might be experimentally observable.

\section{References}

[1] G. Agakishiev, et al., Phys. Rev. Lett. 103 (2009) 132301; Phys. Rev. Lett. 114 (2015) 212301.

[2] A. Angelopoulos, et al., Physics Reports 374 (2003) 165.

[3] C. Patrignani et al., (Particle Data Group) Chin. Phys. C40 (2016) 100001.

[4] A. Aaij, et al., (LHCb Collaboration) Phys. Rev. Lett. 110 (2013) 101802.

[5] C. Hartnack, et al., Physics Reports 510 (2012) 119.

[6] P.H. Eberhard, F. Uchiyama, Nucl. Inst. Meth. Phys. Res. A350 (1994) 144.

[7] C.G. Branco, L. Lavoura, J.P. Silva, CP Violation, Clarendon Press, Oxford (1999).

[8] G. Amelino-Camelia and J. Kapusta, Phys. Lett. B465 (1999) 291.

[9] L.S. Littenberg, Phys. Rev. D21 (1980) 2027. 